\title{
openheart Cost-effectiveness of chiropractic care versus self-management in patients with musculoskeletal chest pain
}

\author{
Mette Jensen Stochkendahl, ${ }^{1}$ Jan Sørensen, ${ }^{2}$ Werner Vach, ${ }^{3}$ \\ Henrik Wulff Christensen, ${ }^{1}$ Poul Flemming Høilund-Carlsen, ${ }^{4}$ Jan Hartvigsen ${ }^{1,5}$
}

To cite: Stochkendahl MJ, Sørensen J, Vach W, et al. Cost-effectiveness of chiropractic care versus selfmanagement in patients with musculoskeletal chest pain. Open Heart 2016;3:e000334. doi:10.1136/openhrt-2015000334

- Additional material is available. To view please visit the journal (http://dx.doi.org/ 10.1136/openhrt-2015000334).

Received 8 September 2015 Revised 22 October 2015 Accepted 5 December 2015

CrossMark

For numbered affiliations see end of article.

Correspondence to Dr Mette Jensen Stochkendahl; m.jensen@nikkb.dk

\section{ABSTRACT}

Aims: To assess whether primary sector healthcare in the form of chiropractic care is cost-effective compared with self-management in patients with musculoskeletal chest pain, that is, a subgroup of patients with nonspecific chest pain.

Methods and results: 115 adults aged 18-75 years with acute, non-specific chest pain of musculoskeletal origin were recruited from a cardiology department in Denmark. After ruling out acute coronary syndrome and receiving usual care, patients with musculoskeletal chest pain were randomised to 4 weeks of communitybased chiropractic care $(n=59)$ or to a single information session aimed at encouraging selfmanagement as complementary to usual care $(n=56)$. Data on resource use were obtained from Danish national registries and valued from a societal perspective. Patient cost and health-related qualityadjusted life years (QALYs; based on EuroQol fivedimension questionnaire (EQ-5D) and Short Form 36item Health Survey (SF-36)) were compared in costeffectiveness analyses over 12 months from baseline. Mean costs were $€ 2183$ lower for the group with chiropractic care, but not statistically significant (95\% $\mathrm{Cl}-4410.5$ to 43.0$)$. The incremental cost-

effectiveness ratio suggested that chiropractic care was cost-effective with a probability of $97 \%$, given a threshold value of $€ 30000$ per QALY gained. In both groups, there was an increase in the health-related quality of life, and the mean increases were similar over the 12-month evaluation period. The mean differences in QALYs between the groups were negligible.

Conclusions: Chiropractic care was more costeffective than self-management. Therefore, chiropractic care can be seen as a good example of a targeted primary care approach for a subgroup of patients with non-specific chest pain.

Trial registration number: NCT00462241.

\section{INTRODUCTION}

Over the past 25 years, hospital admissions for suspected acute coronary syndrome have increased in many countries. ${ }^{12}$ The majority of these patients are diagnosed with angina pectoris or as suffering from non-specific

\section{KEY QUESTIONS}

What is already known about this subject?

- Patients with non-specific chest pain feel equally or more disabled than patients with cardiac chest pain and are a major burden on healthcare resources

- Patients with non-specific chest pain are an established subset of patients who present to chest pain units, and musculoskeletal problems are well recognised as a potential cause of chest pain.

- There are few interventions that offer care in a cost-effective manner for this patient group.

What does this study add?

- In terms of health-related quality of life, primary care in the form of chiropractic care has similar effectiveness as self-management in patients with musculoskeletal chest pain.

- Community-based chiropractic care is more cost-effective than self-management as it is associated with fewer hospital admissions and lower healthcare costs.

How might this impact on clinical practice?

- The results indicate that close contact with a primary care provider lowers the rate of hospitalisation, which is not achieved when patients are left to manage their condition on their own. A multifaceted, person-centred approach targeting both psychosocial and biomechanical aspects of chest pain is recommended.

chest pain (NSCP), that is, coronary disease is not the cause of their pain. NSCP may account for up to $80 \%$ of visits to rapid access chest pain clinics because of new episodes of chest pain. ${ }^{34}$

In 2013, the mean annual societal cost per NSCP patient was estimated at $€ 10000 .^{5}$ Given that 15 million patients are admitted to a hospital with NSCP annually in the USA and Europe ${ }^{6}{ }^{7}$ the total costs exceed $€ 150$ billion annually, which is similar to the costs incurred by society for patients diagnosed with acute myocardial infarction and angina 
pectoris. ${ }^{5} 689$ Hospital admission days account for 70 $90 \%$ of the initial healthcare costs, ${ }^{6}{ }^{10}$ but patients with NSCP continue to incur costs because they often experience repeated episodes of pain, ${ }^{11}{ }^{12}$ that result in further admissions, ${ }^{58}$ decreased quality of life, ${ }^{11}{ }^{12}$ sick leave and lost productivity. ${ }^{5}$ In addition, they seek care from a range of healthcare providers outside the hospital setting ${ }^{3} 5^{12} 13$ at a level that exceeds those of patients who are diagnosed with chest pain associated with cardiac disease. ${ }^{12}$ The potential benefit of primary care sector healthcare and the related costs for this patient group are rarely investigated in randomised trials. ${ }^{14}$

Patients with NSCP are an established subset of patients who present to chest pain units, and musculoskeletal problems are well recognised as potential causes of chest pain, with an estimated prevalence of 10$30 \%^{15-17}$ among patients with NSCP. In 2012, we reported clinical outcomes of a pragmatic, randomised clinical trial evaluating the relative effectiveness of usual care in combination with a primary sector care approach (chiropractic care) compared with usual care in combination with a minimal intervention (self-management). This was in patients presenting to a Danish university hospital with an episode of acute chest pain that was first diagnosed as NSCP and subsequently diagnosed as musculoskeletal chest pain. ${ }^{18} 19$ The chiropractic care group did significantly better than the self-management group, in terms of global perceived effect after 4 weeks of treatment and pain intensity after 12 weeks; however, there were no significant differences between groups after 52 weeks. The aim of the current analysis was to evaluate the 1-year cost-effectiveness of the two approaches in terms of healthcare utilisation, medication and quality of life using self-report questionnaires and Danish national registry-based resource use data.

\section{METHODS}

The design and methods of the randomised clinical trial have previously been reported. ${ }^{18-20} \mathrm{~A}$ brief overview is provided here.

\section{Settings and participants}

The trial was conducted in an emergency cardiology department at a Danish 1200-bed, urban, university hospital and at four community-based chiropractic clinics from 6 August 2006 to 31 March 2008. The study was approved by the Regional Ethics Committee of Vejle and Funen Counties, Denmark (approval number \#VF 20060002) and registered at ClinicalTrials.gov (identification number NCT00462241). All patients presenting at the emergency unit with an episode of acute chest pain underwent the routine diagnostic procedures performed by specialist cardiology nurses under cardiologist supervision. When a patient was discharged from the unit, the study clinician (MJS) screened the patient's

\section{Box 1 Eligibility criteria}

\section{Inclusion criteria}

- Participants should have had a primary complaint of acute chest pain of $<7$ days' duration, but not have had a diagnosis of acute coronary syndrome or another definite cardiac or medical diagnosis causing the chest pain, should be aged 1875 years, be a resident of the local county and be able to read and understand Danish. In addition, participants should have undergone diagnostic procedures to rule out acute coronary syndrome and should not have shown significant comorbidity or contraindications for spinal manipulative therapy.

\section{Exclusion criteria}

- Previous acute coronary syndrome, prior percutaneous coronary intervention or coronary artery bypass grafting, inflammatory joint disease, insulin-dependent diabetes, fibromyalgia, malignant disease, major osseous anomaly, osteoporosis, apoplexy or dementia, inability to cooperate, and pregnancy. In each case, the cause of exclusion was noted.

records to assess eligibility for inclusion in the trial. The eligibility criteria are presented in box 1 .

\section{Trial procedures}

After providing written informed consent, the participants were assessed at baseline by the study clinician using a standardised and previously validated examination protocol. ${ }^{20-22}$ The baseline examination protocol consisted of four main components: patient self-report questionnaires, a detailed case history, a general health examination, and systematic palpation of the chest wall and spine. Patients were classified as having musculoskeletal chest pain if the pain was deemed to be caused by mechanical joint and muscle dysfunction related to C4-T8 somatic structures of the spine and chest wall as established by palpation. ${ }^{20}$ Only patients with a diagnosis of musculoskeletal chest pain were eligible for randomisation. Demographic and clinical information was collected through patient self-report questionnaires and checklists used by the study clinician. Detailed trial procedures, sample size estimation, randomisation and blinding have been described elsewhere. ${ }^{18-20}$

\section{Description of interventions}

At the time of discharge from the Department of Cardiology, both groups received usual care, which typically consisted of a brief ( $5 \mathrm{~min})$ consultation with the attending cardiologist. In the case of persistent or recurrent pain, the patients were instructed to consult their general practitioner (GP).

Participants were randomised into one of two management strategies: chiropractic care or self-management.

\section{Chiropractic care}

Chiropractic consultations were offered as musculoskeletal healthcare management in the primary care sector. In Denmark, chiropractors are an integrated part of the primary healthcare sector and licensed to act as first 
healthcare entry for patients with musculoskeletal symptoms under the Danish National Health Insurance system.

Participants in the chiropractic care group were assigned to one of eight experienced chiropractors in their local community. Each chiropractor applied a pragmatic, individually tailored management strategy based on the age, physical condition and particular problem of each patient. A wide range of manual therapies and exercises were given, adapted to each individual patient. ${ }^{18}$ Furthermore, time was dedicated to address the patient's concerns and to give advice. A maximum of 1020 min treatment sessions one to three times per week for 4 weeks was allowed. If the patient became pain-free sooner, the treating chiropractor could discharge the patient.

\section{Self-management}

Immediately following allocation to the self-management group, the study clinician (MJS) gave each participant a 15 min consultation consisting of reassurance and advice to address the patient's concerns and to promote selfmanagement. Participants were told that their chest pain had a benign, self-limiting course and were given two to three home exercises.

\section{Healthcare utilisation, medication and associated costs}

The analysis was conducted from a societal perspective, that is, all relevant costs and effects were measured regardless of who paid the costs and who benefited from the effects. We included direct costs and effects. The included categories of costs are presented in figure 1 . Danish national registries use unique, personal identification numbers issued to all Danish residents to record data about hospital contacts and primary sector healthcare utilisation. ${ }^{23-25}$ In Denmark, the cost of healthcare utilisation is primarily financed by the Danish National Health Service. Exceptions to this are chiropractic, physiotherapy and psychology consultations, which incur some additional out-of-pocket expenses paid by the patient. Use of prescriptive medication is registered in the Odense Pharmaco-Epidemiological Database using international Anatomical Therapeutic Chemical Classification System codes (ATC), which contain information on the dispensing of all medications prescribed by a physician and subsidised by the national health insurance at any pharmacy. ${ }^{26}$ Patient self-report questionnaires about clinical outcomes and resource use that were not available from the national registries were collected at baseline and 4, 12 and 52 weeks after baseline. Patients were asked to describe their use of complementary and alternative medicine (CAM) and nonprescriptive drugs during the previous 3 months.

Direct costs included: the intervention costs (chiropractic care and the self-management sessions); additional visits to mainstream healthcare and CAM providers; prescriptive and non-prescriptive drugs; and hospital contacts. Valuation of a chiropractic intervention was based on standard fees according to the
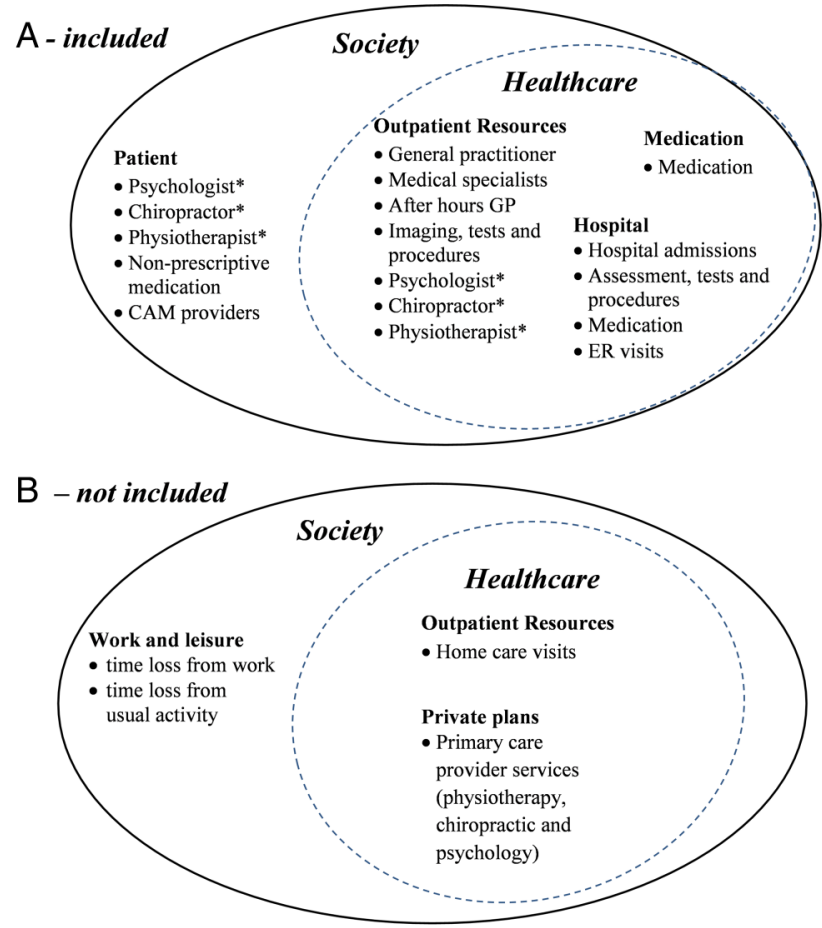

Figure 1 (A) The types of costs that were included in the analysis, and (B) the types of costs that were not included (modified from Torrance et $a^{P^{7}}$ ). *Indicates that the service is paid by the patient, but partially reimbursed by the National Danish Health insurance. CAM, complementary and alternative medicine; ER, emergency room; GP, general practitioner.

national collective agreement between the Danish Chiropractic Association and the Danish National Health Service. A self-management session was valued as being equivalent to one standard chiropractic consultation. Costs of prescriptive medication were calculated on the basis of prices charged by the pharmacies (excluding VAT), and the costs of primary healthcare provider and hospital contacts were based on the standard patient fees paid by the Danish National Health Service. All monetary values are presented in euros at 2014 cost levels, and therefore costs were uplifted to August 2014 price levels using the Consumer Price Index maintained by Statistics Denmark.

\section{Health-related quality of life measures}

General health status was measured using the Medical Outcomes Study Short Form 36-item Health Survey (SF-36, v1US). The SF-36 is a generic health status instrument covering both physical and mental aspects of health. ${ }^{28}{ }^{29}$ The six-dimensional health status short form (SF-6D) is a health status classification system that can be generated from the SF-36 responses. It is composed of six multilevel dimensions: physical functioning, role limitations, social functioning, pain, mental health and vitality. A scoring programme has been developed to transform the SF-36 responses into a single preference- 
weighted quality-of-life index (QoL-index) score (SF-6D); the revised Brazier algorithm being used here to score the SF-36 data into the score index on a $0-1$ scale. ${ }^{30}$ The algorithm provided scores ranging from 0.160 to $1.0 .^{3132}$

The EuroQol five-dimension questionnaire (EQ-5D) is a generic utility-based health status instrument comprising five dimensions of health (mobility, self-care, usual activities, pain/discomfort, anxiety/depression), which in its original version are each divided into three levels of severity. ${ }^{33}{ }^{34}$ In this study, we used five levels of severity in each dimension, as suggested by Kind and Macran $^{35}$ and subsequently tested by Janssen et al. ${ }^{36} 37$ Patients were asked to describe their health status as of 'today'. The recommended Danish scoring algorithm was applied to calculate an index score, with 0 denoting death and 1 denoting perfect health. ${ }^{38}$ This algorithm provided scores ranging from -0.624 to 1.0 . The EQ-5D instrument includes health states worse than death and assigns to these values lower than 0 (negative values). ${ }^{39}$

\section{Statistical analysis}

Baseline characteristics were reported as percentages for binary variables, and as mean values and SDs for continuous variables. Multiple imputations based on fully conditional specifications were used to impute missing outcome data at follow-up. For each analysis, the imputations were based on a model that included the outcome variables at all time points and group allocation. By multiple imputation, five imputed data sets were created, each of which was analysed separately. The results of the five analyses were pooled using Rubin's rules. ${ }^{40}$

Mean values and SDs were computed for each of the two QoL-index outcome measures in each treatment group. The difference in mean values was assessed using change scores adjusted for baseline differences using analysis of covariance and reported with 95\% CIs. The incremental health-related quality of life (HRQoL) was calculated as the mean difference-in-difference in the QoL-index between the intervention groups. Quality-adjusted life years (QALYs) were also calculated based on the two QoL-indices. The 1-year QALYs were calculated by multiplying the utilities with the amount of time a patient spent in a particular health state. Transitions between health states were linearly interpolated.

Healthcare utilisation was reported as the number and proportion of patients visiting a healthcare provider group or using a service, total and mean number of visits or services, and associated total and mean costs. The two groups were compared at follow-up using $\chi^{2}$ test for proportions and t test (two-sided) for total and mean number of visits. Differences in mean costs per user and per patient in the intervention groups, respectively, were computed using pairwise comparisons of means and reported together with $95 \%$ CIs.

To facilitate comparison between self-report and registry-based costs, costs from the self-report questionnaires were extrapolated to estimate annual costs. Data from the 12 weeks follow-up were extrapolated forwards to estimate the first 26 weeks, and data from the 12-month follow-up were extrapolated backwards to estimate the last 26 weeks.

The average costs per person and treatment effects, as well as the incremental cost-effectiveness ratio between the two groups were calculated and graphically displayed in the cost-effectiveness plane using bias-corrected bootstrapping with 1000 repetitions. Acceptability curves were constructed to show the probability that the chiropractor intervention was cost-effective at different threshold values for a QALY. The probability that the intervention was cost-effective compared with the control was reported for a given maximum acceptable ceiling ratio of $€ 30000$ per QALY and $€ 100000$ per QALY.

These statistical analyses were performed on the basis of the intention-to-treat principle, that is, patients were analysed in the group to which they were allocated. Analyses were performed using STATA (Stata Statistical Software: release V.13.1. Stata Corp, College Station, Texas, USA). Transformation of the SF-36 to the QoL-index was performed using IBM SPSS Statistics release V.21.0.0.0.

\section{RESULTS}

One hundred and fifteen patients were included and randomised, 59 patients to chiropractic care, and 56 to selfmanagement. Randomisation resulted in two groups with similar baseline values for the clinical variables. In the chiropractic care group, $\mathrm{n}=0(0 \%), \mathrm{n}=8(14 \%)$ and $\mathrm{n}=9$ $(15 \%)$ were lost to self-report follow-up at 4,12 and 52 weeks, compared with $n=9(16 \%), n=8(14 \%)$ and $n=16$ $(29 \%)$ in the self-management group. All 115 patients $(100 \%)$ were followed over 1 year using national registries. The flow of participants through the trial is provided in online supplementary figure S1. Table 1 shows the baseline characteristics of the chiropractic care and selfmanagement groups.

\section{Clinical outcomes}

Table 2 presents the HRQoL scores at baseline and at the three follow-up time points. In both groups, there was an increase in the HRQoL scores, but there were no statistically significant differences between the two groups at any of the time points or any trends in favour of either group. The group difference in QALYs was negligible.

\section{Healthcare utilisation and medication}

Online supplementary table S1 lists the healthcare utilisation and associated costs in the chiropractic care and self-management groups. As part of the intervention, the chiropractic care patients had an average of 7.0 chiropractic visits (minimum=1, maximum=10), and 19 patients $(32 \%)$ had an X-ray performed. All patients in 
Table 1 Baseline patient characteristics. Data are expressed as means and \pm SDs or absolute numbers and relative frequencies (in parentheses)

\begin{tabular}{|c|c|c|}
\hline & $\begin{array}{l}\text { Chiropractic } \\
\text { care } \\
n=59\end{array}$ & $\begin{array}{l}\text { Self-management } \\
n=56\end{array}$ \\
\hline Age, years $\pm S D$ & $51.4 \pm 10.0$ & $50.8 \pm 12.1$ \\
\hline Female, n (\%) & $26(44.0)$ & 22 (39.3) \\
\hline $\begin{array}{l}\text { Maximum chest pain } \\
(0-10),{ }^{*} \pm S D\end{array}$ & $6.7 \pm 2.4$ & $5.9 \pm 2.3$ \\
\hline EQ-5D, mean $\pm S D$ & $0.738 \pm 0.021$ & $0.718 \pm 0.022$ \\
\hline SF-6D, mean $\pm S D$ & $0.695 \pm 0.015$ & $0.676 \pm 0.016$ \\
\hline $\begin{array}{l}\text { Married/living with } \\
\text { someone, n (\%) }\end{array}$ & $51(86.4)$ & $50(90.9)$ \\
\hline Working, n (\%) & $44(75.9)$ & $35(63.6)$ \\
\hline College graduate, $\mathrm{n}(\%)$ & $16(27.6)$ & $16(30.2)$ \\
\hline \multicolumn{3}{|l|}{ Expectation of treatment $\dagger$} \\
\hline Chiropractic care $\pm S D$ & $2.2 \pm 0.5$ & $2.1 \pm 0.6$ \\
\hline Self-management $\pm S D$ & $2.3 \pm 0.5$ & $2.3 \pm 0.6$ \\
\hline \multicolumn{3}{|c|}{$\begin{array}{l}{ }^{*} \text { Pain intensity was reported on an } 11-\text { point numeric rating scale } \\
\text { ranging from } 0=\text { no pain to } 10=\text { worst possible pain. } \\
\dagger \text { Expectation was reported as average ratings on a five-point box } \\
\text { scale (much better=1 to much worse }=5 \text { ). } \\
\text { EQ-5D, EuroQol five-dimension questionnaire; SF-6D, } \\
\text { six-dimensional health status short form. }\end{array}$} \\
\hline
\end{tabular}

the self-management group $(\mathrm{n}=56)$ completed the information session. Overall, fewer chiropractic care patients used healthcare services and those that did had a lower mean number of visits for some of these services compared with the self-management patients: GP after-hours service ( $12 \%$ vs $29 \%$ ( $p=0.03), 1.1$ vs 2.0 visits $(p=0.11)$ ), admissions to cardiology departments (3\% vs $18 \%$ $(\mathrm{p}=0.01), 1.0$ vs 1.3 admissions $(\mathrm{p}=0.28))$, cardiology ambulatory visits $(14 \%$ vs $30 \%$ ( $\mathrm{p}=0.03), 1.1$ vs 1.5 visits $(\mathrm{p}=0.13))$ and other ambulatory visits $(34 \%$ vs $41 \%$ $(\mathrm{p}=0.43), 2.3$ vs 4.0 visits $(\mathrm{p}=0.02)$ ). For visits to primary care musculoskeletal providers (chiropractors and physiotherapists), the chiropractic care group showed a trend towards a higher proportion of patients visiting chiropractors $(32 \%$ vs $18 \%$ ( $\mathrm{p}=0.08), 4.6$ vs 5.8 visits $(\mathrm{p}=0.07))$ in the follow-up period compared with the self-management group, but a slightly lower proportion visiting physiotherapists $(10 \%$ vs $14 \% \quad(p=0.50), 5.8$ vs 35.1 visits $(\mathrm{p}=0.27))$. For both provider types, the chiropractic care group had a statistically non-significant lower mean number of visits. Use of non-prescriptive and prescriptive medication was similar for the two groups, except for non-prescriptive mild analgesics $(32 \%$ vs $13 \%(\mathrm{p}=0.01))$ and herbal medicine $(15 \%$ vs $0 \%$ $(\mathrm{p}=0.002)$, which were significantly higher in the chiropractic care group, and lower for prescriptive opioids (7\% vs $13 \%(\mathrm{p}=0.3))$ and lipid-modifying drugs $(9 \%$ vs $17 \%$ ( $\mathrm{p}=0.05$ ); see online supplementary table S2).

\section{Costs}

The mean intervention cost of the chiropractic care was higher than the cost of self-management ( $€ 403.7$ vs $€ 44.8$, mean difference $€ 358.9$, 95\% CI (321.4 to 396.4); see online supplementary tables S1 and S4). Subsequent healthcare costs relating to primary care were overall similar between the groups ( $€ 409.4$ vs $€ 502.9$, mean difference $-€ 93.5 ; 95 \%$ CI $(-321.6$ to 134.7$)$ ), whereas costs of hospital care were lower in the chiropractic care group ( $€ 1919.5$ vs $€ 4305.8$, mean difference $-€ 2386.3$, $95 \%$ CI $(-4580.5$ to -192.0$))$.

Total 1-year costs in the chiropractic care group were $€ 2183$ lower than in the self-management group, but this difference was not statistically significant $(95 \%$ CI ( -4410.5 to 43.0$)$; table 3$)$. The main contributor to the cost difference was admissions to hospital (see online supplementary table $\mathrm{S} 1$ ).

\section{Cost-effectiveness}

The incremental costs were $-€ 2184$ (savings) and the incremental effect was 0.0092 and 0.0096 QALY using the EQ-5D and SF-36, respectively. This indicates that the chiropractor intervention dominated the information intervention by providing similar (or slightly improved) health utility at an overall lower cost.

The joint distribution of costs and effects (EQ-5D) are presented in figure 2. Sixty-five per cent of the bootstrapped incremental cost-effect pairs fall in the southeast quadrant indicating a positive QALY gain at lower costs by chiropractic care compared with selfmanagement. Thirty-three per cent falls in the southwest quadrant, indicating less health utility but at lower costs. Consequently, there is a $65 \%$ probability that chiropractic care generates better outcomes and is less costly than self-management. These findings are similar when the SF-36 was used as the utility measure (data not shown). The cost-effectiveness acceptability curve using EQ-5D is presented in online supplementary figure S2. Given a maximum acceptable ceiling ratio of $€ 30000$ per QALY gained, the probability that chiropractic care is costeffective compared with self-management is 0.972 . At $€ 100000$ per QALY gained, the probability is 0.881 . When using SF-36, the probability that chiropractic care is cost-effective compared with self-management is 0.958 and 0.874 given a maximum acceptable ceiling ratio of $€ 30000$ and $€ 100000$ per QALY gained, respectively (data not shown).

\section{DISCUSSION}

In this analysis of data from a randomised clinical trial assessing the cost-effectiveness of two strategies to reduce pain and improve quality of life in patients with musculoskeletal chest pain, we observed no statistically significant differences between the two strategies in QALYs gained. We did, however, observe lower total healthcare costs in the chiropractic care group during the first year after enrolment in the trial $(-€ 2184 ; 95 \%$ CI -4411 to 43). Assuming identical gains in HRQoL, the study showed that chiropractic care is more costeffective when compared with self-management in patients presenting with an acute episode of 
Table 2 Multiple imputed clinical outcomes at follow-up

\begin{tabular}{|c|c|c|c|}
\hline Outcome & $\begin{array}{l}\text { Chiropractic care } \\
(n=59) \\
\text { Mean (SD) }\end{array}$ & $\begin{array}{l}\text { Self-management } \\
(\mathrm{n}=56) \\
\text { Mean (SD) }\end{array}$ & $\begin{array}{l}\text { Difference* }^{*} \\
\Delta(95 \% \mathrm{Cl})\end{array}$ \\
\hline \multicolumn{4}{|l|}{ EQ-5D } \\
\hline Baseline & $0.738 \pm 0.021$ & $0.718 \pm 0.022$ & \\
\hline 4 weeks & $0.786(0.021)$ & $0.796(0.018)$ & $-0.021(-0.065$ to 0.023$)$ \\
\hline 12 weeks & $0.811(0.023)$ & $0.792(0.020)$ & $0.009(-0.044$ to 0.062$)$ \\
\hline 52 weeks & $0.826(0.023)$ & $0.823(0.024)$ & $-0.033(-0.069$ to 0.062$)$ \\
\hline 1-year QALY (EQ-5D) & $0.811(0.019)$ & $0.802(0.017)$ & 0.0094 ( -0.041 to 0.060$)$ \\
\hline \multicolumn{4}{|l|}{ SF-36 } \\
\hline Baseline & $0.695 \pm 0.015$ & $0.676 \pm 0.016$ & \\
\hline 4 weeks & $0.742(0.018)$ & $0.727(0.020)$ & $0.004(-0.039$ to 0.047$)$ \\
\hline 12 weeks & $0.757(0.018)$ & $0.754(0.021)$ & $-0.010(-0.056$ to 0.035$)$ \\
\hline 52 weeks & $0.788(0.018)$ & $0.774(0.021)$ & $0.003(-0.045$ to 0.051$)$ \\
\hline 1-year QALY (SF-6D) & $0.765(0.0157)$ & $0.756(0.0190)$ & $0.0092(-0.039$ to 0.058$)$ \\
\hline
\end{tabular}

${ }^{*}$ The mean outcome differences between chiropractic care and self-management were adjusted for the baseline difference.

EQ-5D, EuroQol five-dimension questionnaire; QALY, quality-adjusted life year; SF-6D, six-dimensional health status short form; SF-36, Short Form 36-item Health Survey.

musculoskeletal chest pain. Similar results, when using the two utility measures, support the robustness of these findings. The study showed that this patient group with musculoskeletal chest pain has a high use of healthcare resources with high associated costs.

Using registry-based data on healthcare utilisation, prescriptions and associated costs have two major advantages. First, using the unique personal identification number assigned to each Danish resident, we were able to track all hospital and public primary care utilisation and medication use over the 1-year follow-up period with no loss to follow-up. We were not able to track the use of primary care utilisation covered by private insurance plans (primarily use of chiropractic, physiotherapy and psychology services), but compared with hospital costs, the costs of these private plans are negligible and not likely to influence the results. Second, the presented costs are actual incurred costs, which reduced the uncertainty regarding the cost estimates. Compared with Swedish reports, ${ }^{5}$ annual average costs were lower in our study (€3000-€5200); however, this difference may be explained by the lower mean age in our study or perhaps the subset of patients with NSCP suffering from musculoskeletal chest pain incurs fewer costs compared with a mixed group with NSCP. We did not take into account indirect costs, such as sick leave and lost productivity. According to Mourad et al, ${ }^{5}$ costs of sick leave, disability pension and reduced productivity are approximately one-third of total costs. This means that future research into NSCP interventions should address not only healthcare costs, but also how the intervention impacts on work life.

The two groups reported similar HRQoL at all time points, and they were all clinically relevant improvements that were above the minimal important difference ${ }^{41}$ for the EQ-5D and SF-36 scores, with the largest improvement after 4 weeks. Despite these improvements, both groups reported HRQoL outcomes below the age-matched and sex-matched Danish population norms ${ }^{42}$ at baseline and at all three follow-ups which confirm that NSCP has an important impact on psychological health and quality of life. ${ }^{43}$ The statistically significant difference in clinical improvement (pain intensity and global perceived effect) in favour of the chiropractic care group seen in the previous reports of this clinical trial ${ }^{18} 19$ was not reflected in the HRQoL measures. This may indicate that the two interventions provided similar effects in HRQoL in patients with musculoskeletal chest pain. Alternatively, it could be that the HRQoL measures, which were originally designed to measure change in chronic conditions, are not sufficiently responsive to reflect change in pain intensity or reflect those changes that caused these patients to report a global effect in this acute chest pain condition. This study was powered to detect a difference in the primary outcomes (maximum chest pain and global perceived effect at 4 weeks). It is likely that the study is underpowered to

Table 3 The total and mean costs of the interventions, healthcare utilization and 1 -yer total costs per person $(€)$ with $95 \% \mathrm{Cls}$

\begin{tabular}{|c|c|c|c|c|c|}
\hline & \multicolumn{2}{|c|}{$\begin{array}{l}\text { Chiropractic care } \\
n=59\end{array}$} & \multicolumn{2}{|c|}{$\begin{array}{l}\text { Self-management } \\
n=56\end{array}$} & \multirow{2}{*}{$\begin{array}{l}\text { Mean difference } \\
\Delta(95 \% \mathrm{Cl})\end{array}$} \\
\hline & Total costs & $\begin{array}{l}\text { Mean costs per patient } \\
(95 \% \mathrm{Cl})\end{array}$ & Total costs & $\begin{array}{l}\text { Mean costs per patient } \\
(95 \% \mathrm{Cl})\end{array}$ & \\
\hline Intervention costs & 23821 & 404 (367 to 441$)$ & 2511 & 45 (45 to 45$)$ & 359 (321 to 396$)$ \\
\hline Healthcare costs & 155604 & 2637 (1942 to 3333 ) & 290082 & 5180 (2990 to 7370$)$ & $-2543(-4767$ to -319$)$ \\
\hline Total 1-year costs & 179426 & 3041 (2337 to 3745$)$ & 292593 & 5225 (3035 to 7415$)$ & -2184 ( -4411 to 43$)$ \\
\hline
\end{tabular}




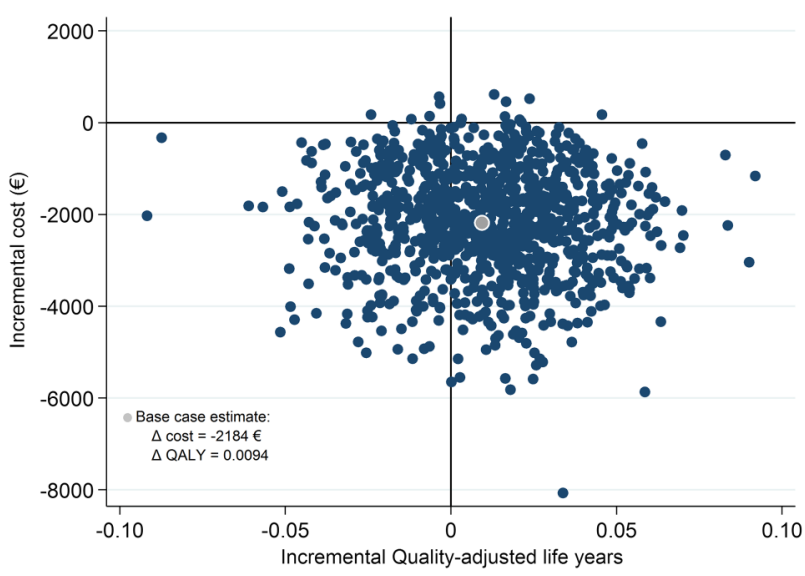

Figure 2 Scatter plot of estimated joint density of incremental costs and incremental effects (quality-adjusted life years (EuroQol five-dimension questionnaire, EQ-5D)) of chiropractic care versus self-management obtained by bootstrap resampling.

detect a difference in HRQoL at 1-year follow-up with the accompanying risk of a type 2 error. Owing to the limited follow-up of 1 year, we were not able to observe potentially long-term adverse effects of reduced hospital admission in the chiropractic care group; however, we have previously shown that the risk of future cardiac events is low in patients with NSCP and will likely occur within the first year after hospitalisation, if at all. ${ }^{44}$

This study was designed as a pragmatic trial with a 12-month follow-up period that was conducted in the real-life context of a large university hospital in Denmark and four community-based chiropractic practices, which strengthens the external validity of our results. ${ }^{45}$ The main contributor to the lower cost of the chiropractor care group was the lower rate of hospitalisation in that group. We are not able to elucidate why, but psychological factors frequently influence healthcareseeking generally and have been suggested in chest pain patients specifically. ${ }^{46}$ In particular, fear of activities that cause physical (somatic) sensation with normal activities and 'normal' discomfort (interoceptive fear) are an important factor in understanding the persistent medical-seeking behaviours of some patients with NSCP. $^{43} 47 \quad 48$ We suggest that the key factors in the success of the chiropractic care were the close contact with a primary healthcare provider, who targeted unmet distress, the consideration of psychological and physical factors together, and the neurological and analgesic effect of manual therapy. In any case, it is clear that offering a series of consultations with a musculoskeletal healthcare provider may have positive economic implications that are not achieved when patients are left to manage their condition on their own.

NSCP is defined as the absence of coronary disease and is not a unitary diagnosis but contains a number of diverse subgroups. ${ }^{3}$ Patients with NSCP may have evidence of gastro-oesophageal, musculoskeletal, psychiatric or psychological abnormalities, and signs of one or more co-occurring conditions. It has been argued that the ability to provide adequate care is limited by a lack of information on the characteristics of patients with NSCP. ${ }^{3}$ By means of a systematic clinical assessment of this patient group, we were able to identify a subset of patients with suspected musculoskeletal chest pain, and we have demonstrated the cost-effectiveness of a safe and low-tech management strategy for this specific patient group. Considering the large number of people with NSCP, a recommendation in future clinical guidelines of primary care treatment for musculoskeletal chest pain from clinicians, such as chiropractors or similar professions, could result in considerable savings to societies, provided that a multifaceted, person-centred approach was used. In our clinical trial, that comprehensive approach included manual treatment, exercises and advice targeting both psychosocial and biomechanical aspects of chest pain. Future research should evaluate the generalisability of our results to other settings, look into understanding the potentially multifactorial causes of NSCP and recognise the potential for multiple coexisting and potentially mutually reinforcing causes, in diagnosis and treatment.

\section{CONCLUSION}

We observed no significant difference in HRQoL, but we did observe significant cost-savings relating to hospital contacts in patients who received usual care in combination with community-based chiropractic care compared with usual care and self-management. This suggests that offering a series of consultations with a musculoskeletal healthcare provider is a more cost-effective approach compared with leaving patients to manage the condition themselves.

\section{Author affiliations}

${ }^{1}$ Nordic Institute of Chiropractic and Clinical Biomechanics, Odense M, Denmark

${ }^{2}$ Department of Public Health, Centre for Health Economics Research (COHERE), University of Southern Denmark, Odense C, Denmark ${ }^{3}$ Center of Medical Biometry and Medical Informatics, Institute of Medical Biometri and Medical Informatics, University Medical Center Freiburg, Freiburg, Germany

${ }^{4}$ Department of Nuclear Medicine, Odense University Hospital, Odense C, Denmark

${ }^{5}$ Institute of Sports Science and Clinical Biomechanics, University of Southern Denmark, Odense C, Denmark

Acknowledgements The authors thank Eleanor Boyle, PhD, for help with the transformation of the SF-36 to the QoL-index and for statistical advice.

Contributors MJS, WV, HWC, PFH-C and JH contributed to the concept and design of the clinical components of the study, which was led by MJS. MJS, JS and JH developed the economic component of the study. MJS, JS and WV developed the analytic plan for the study. MJS and JS analysed its results. MJS drafted the manuscript and all authors critically revised the article for important intellectual content and gave final approval of the version to be published. MJS is the guarantor. All authors had full access to all of the data in the study and take responsibility for the integrity of the data and the accuracy of the data analysis.

Funding This research was partly funded by the Region of Southern Denmark (former Funen County), the Danish Chiropractic Foundation, and the Scott Haldeman Award.

Competing interests None declared. 
Ethics approval Obtained from the Regional Ethics Committee of Vejle and Funen Counties, Denmark (approval number \#VF 20060002), the Danish Data Protection Agency (journal number \#2013-41-2012) and registered at ClinicalTrials.gov (identification number NCT00462241). All participants gave written informed consent.

Provenance and peer review Not commissioned; internally peer reviewed.

Data sharing statement No additional data are available.

Open Access This is an Open Access article distributed in accordance with the Creative Commons Attribution Non Commercial (CC BY-NC 4.0) license, which permits others to distribute, remix, adapt, build upon this work noncommercially, and license their derivative works on different terms, provided the original work is properly cited and the use is non-commercial. See: http:// creativecommons.org/licenses/by-nc/4.0/

\section{REFERENCES}

1. Fagring AJ, Lappas G, Kjellgren $\mathrm{KI}$, et al. Twenty-year trends in incidence and 1-year mortality in Swedish patients hospitalised with non-AMI chest pain. Data from 1987-2006 from the Swedish hospital and death registries. Heart 2010;96:1043-9.

2. Murphy NF, Maclntyre K, Capewell S, et al. Hospital discharge rates for suspected acute coronary syndromes between 1990 and 2000 : population based analysis. BMJ 2004;328:1413-14.

3. Marks EM, Chambers JB, Russell V, et al. The rapid access chest pain clinic: unmet distress and disability. QJM 2014;107:429-34.

4. Goodacre S, Cross E, Arnold J, et al. The health care burden of acute chest pain. Heart 2005;91:229-30.

5. Mourad G, Alwin J, Strömberg A, et al. Societal costs of non-cardiac chest pain compared with ischemic heart disease-a longitudinal study. BMC Health Serv Res 2013;13:403.

6. Groarke J, O'Brien J, Go G, et al. Cost burden of non-specific chest pain admissions. Ir J Med Sci 2013;182:57-61.

7. Nawar EW, Niska RW, Xu J. National Hospital Ambulatory Medical Care Survey: 2005 emergency department summary. Adv Data 2007;(386):1-32.

8. Leise MD, Locke GR III, Dierkhising RA, et al. Patients dismissed from the hospital with a diagnosis of noncardiac chest pain: cardiac outcomes and health care utilization. Mayo Clin Proc 2010;85:323-30.

9. Tew R, Guthrie EA, Creed FH, et al. A long-term follow-up study of patients with ischaemic heart disease versus patients with nonspecific chest pain. J Psychosom Res 1995;39:977-85.

10. Forberg JL, Henriksen LS, Edenbrandt L, et al. Direct hospital costs of chest pain patients attending the emergency department: a retrospective study. BMC Emerg Med 2006;6:6.

11. Chambers JB, Marks EM, Russell V, et al. A multidisciplinary, biopsychosocial treatment for non-cardiac chest pain. Int J Clin Pract 2015;69:922-7.

12. Eslick GD, Talley NJ. Non-cardiac chest pain: predictors of health care seeking, the types of health care professional consulted, work absenteeism and interruption of daily activities. Aliment Pharmacol Ther 2004;20:909-15.

13. Glombiewski JA, Rief W, Bösner $S$, et al. The course of nonspecific chest pain in primary care: symptom persistence and health care usage. Arch Intern Med 2010;170:251-5.

14. Burgstaller JM, Jenni BF, Steurer J, et al. Treatment efficacy for non-cardiovascular chest pain: a systematic review and meta-analysis. PLoS ONE 2014;9:e104722.

15. Parkash O, Almas A, Hameed A, et al. Comparison of non cardiac chest pain (NCCP) and acute coronary syndrome (ACS) patients presenting to a tertiary care center. J Pak Med Assoc 2009;59:667-71.

16. Knockaert DC, Buntinx F, Stoens N, et al. Chest pain in the emergency department: the broad spectrum of causes. Eur J Emerg Med 2002;9:25-30.

17. Fruergaard $P$, Launbjerg J, Hesse $B$, et al. The diagnoses of patients admitted with acute chest pain but without myocardial infarction. Eur Heart J 1996;17:1028-34

18. Stochkendahl MJ, Christensen HW, Vach W, et al. Chiropractic treatment vs self-management in patients with acute chest pain: a randomized controlled trial of patients without acute coronary syndrome. J Manipulative Physiol Ther 2012;35:7-17.

19. Stochkendahl MJ, Christensen HW, Vach W, et al. A randomized clinical trial of chiropractic treatment and self-management in patients with acute musculoskeletal chest pain: 1-year follow-up. J Manipulative Physiol Ther 2012;35:254-62.
20. Stochkendahl MJ, Christensen HW, Vach W, et al. Diagnosis and treatment of musculoskeletal chest pain: design of a multi-purpose trial. BMC Musculoskelet Disord 2008;9:40.

21. Brunse $\mathrm{MH}$, Stochkendahl MJ, Vach W, et al. Examination of musculoskeletal chest pain-an inter-observer reliability study. Man Ther 2010;15:167-72

22. Christensen HW, Vach W, Gichangi A, et al. Cervicothoracic angina identified by case history and palpation findings in patients with stable angina pectoris. J Manipulative Physiol Ther 2005;28:303-11.

23. Pedersen CB. The Danish Civil Registration System. Scand J Public Health 2011;39(Suppl 7):22-5.

24. Lynge E, Sandegaard JL, Rebolj M. The Danish National Patient Register. Scand J Public Health 2011;39(Suppl 7):30-3.

25. Andersen JS, Olivarius Nde F, Krasnik A. The Danish National Health Service Register. Scand J Public Health 2011;39(Suppl 7):34-7.

26. Gaist D, Sorensen HT, Hallas J. The Danish prescription registries. Dan Med Bulletin 1997;44:445-8.

27. Torrance GW, Raynauld JP, Walker V, et al. A prospective, randomized, pragmatic, health outcomes trial evaluating the incorporation of hylan G-F 20 into the treatment paradigm for patients with knee osteoarthritis (Part 2 of 2): economic results. Osteoarthritis Cartilage 2002;10:518-27.

28. Bjorner JB, Kreiner S, Ware JE, et al. Differential item functioning in the Danish translation of the SF-36. J Clin Epidemiol 1998;51:1189-202

29. Bjorner JB, Damsgaard MT, Watt T, et al. Tests of data quality, scaling assumptions, and reliability of the Danish SF-36. J Clin Epidemiol 1998;51:1001-11.

30. Brazier JE, Rowen D, Hanmer J. Revised SF-6D scoring programmes: a summary of improvements. Patient Reported Outcomes Newsletter. 2008:14-15. http://www.pro-newsletter.com/ images/PDF articles/sf-6d.pdf (accessed 8 Sep 2015).

31. Kharroubi SA, Brazier JE, Roberts J, et al. Modelling SF-6D health state preference data using a nonparametric Bayesian method. $J$ Health Econ 2007;26:597-612.

32. Brazier JE, Roberts J. The estimation of a preference-based measure of health from the SF-12. Med Care 2004:42:851-9.

33. Brooks $\mathrm{R}$, Rabin $\mathrm{R}$, de Charro $\mathrm{F}$. The measurement and valuation of health status using EQ-5D: A European perspective: evidence from the EuroQol BIO MED research programme. Dordrecht, The Netherlands: Kluwer Academic Publishers, 2003.

34. Gudex C, Sørensen J. EQ-SD: A generic health status instrument [in Danish]. Maanedsskrift Praktisk Laegegerning 1998;76:1339-45.

35. Kind $P$, Macran S. A five-level version of EQ-5D. Value Health 2004;7:650

36. Janssen MF, Birnie E, Haagsma JA, et al. Comparing the standard EQ-5D three-level system with a five-level version. Value Health 2008;11:275-84.

37. Janssen MF, Birnie E, Bonsel GJ. Quantification of the level descriptors for the standard EQ-5D three-level system and a five-level version according to two methods. Qual Life Res 2008;17:463-73.

38. Oppe M, Devlin NJ, Szende A. EQ-5D value sets: inventory, comparative review and user guide. Dordrecht, The Netherlands: Springer, 2007.

39. Wittrup-Jensen KU, Lauridsen J, Gudex C, et al. Generation of a Danish TTO value set for EQ-5D health states. Scand J Public Health 2009;37:459-66.

40. Rubin DB. Multiple imputation after 18+ years. J Am Stat Assoc 1996;91:473-89.

41. Walters SJ, Brazier JE. Comparison of the minimally importan difference for two health state utility measures: EQ-5D and SF-6D. Qual Life Res 2005;14:1523-32.

42. Sørensen J, Davidsen M, Gudex C, et al. Danish EQ-5D population norms. Scand J Public Health 2009;37:467-74.

43. Webster R, Norman P, Goodacre S, et al. The prevalence and correlates of psychological outcomes in patients with acute non-cardiac chest pain: a systematic review. Emerg Med $\mathrm{J}$ 2012;29:267-73.

44. Stochkendahl MJ, Mickley $\mathrm{H}$, Vach W, et al. Clinical characteristics, myocardial perfusion deficits, and clinical outcomes of patients with non-specific chest pain hospitalized for suspected acute coronary syndrome: a 4-year prospective cohort study. Int J Cardiol 2015;182:126-31.

45. Ramsey S, Willke R, Briggs A, et al. Good research practices for cost-effectiveness analysis alongside clinical trials: the ISPOR RCT-CEA Task Force report. Value Health 2005; 8:521-33 
46. Mourad G, Strömberg A, Johansson P, et al. Depressive symptoms, cardiac anxiety, and fear of body sensations in patients with non-cardiac chest pain, and their relation to healthcare-seeking behavior: a cross-sectional study. Patient 2016:9:69-77.

47. Hadlandsmyth K, Rosenbaum DL, Craft JM, et al. Health care utilisation in patients with non-cardiac chest pain: a longitudinal analysis of chest pain, anxiety and interoceptive fear. Psychol Health 2013;28:849-61.

48. Robertson N, Javed N, Samani NJ, et al. Psychological morbidity and illness appraisals of patients with cardiac and non-cardiac chest pain attending a rapid access chest pain clinic: a longitudinal cohort study. Heart 2008;94:e12. 